\title{
A MULTIMODE APPROACH TO GEOMETRICALLY NON-LINEAR FORCED VIBRATION OF BEAMS CARRYING POINT MASSES
}

\author{
Hatim FAKHREDDINE ${ }^{1}$, Ahmed ADRI ${ }^{1}$, Mohcine CHAJDI ${ }^{2}$, Said RIFAI ${ }^{1}$, Rhali BENAMAR ${ }^{3}$ \\ ${ }^{1}$ Hassan II University of Casablanca, EST, LMPGI, B.P.8012, Oasis Casablanca, Morocco \\ Hatim.fakhreddine@gmail.com \\ ${ }^{2}$ Mohammed V University in Rabat, ENSET - Rabat, MSSM, B.P.6207, Rabat, Morocco \\ ${ }^{3}$ Mohammed V University in Rabat, EMI-Rabat, LERSIM, Agdal, B.P. 765, Rabat, Morocco
}

\begin{abstract}
The present work deals with the geometrically non-linear forced vibrations of beams carrying a concentric mass under different end conditions. Considering the axial strain energy and expanding the transverse displacement in the form of a finite series of spatial functions, the application of Hamilton's principle reduces the vibration problem to a non-linear algebraic system solved by an approximate method developed previously. In order to validate the approach, comparisons are made of the present solutions with those previously obtained by the finite element method. Focus is made here on the analysis of the non-linear stress distribution in the beam with an attached mass. The non-linear forced deflection shapes and their corresponding curvatures are presented for different magnitudes of the attached mass, different excitation levels and different vibration amplitudes.
\end{abstract}

Keywords: Geometrical non-linearity, Forced vibrations, Multimode approach, Stress distribution, point masses.

\section{INTRODUCTION}

In the structural design, the major concern is to obtain a structure which is light, resistant, and with a very long fatigue life. In this perspective, the analysis of the dynamic behaviour of a structure is essential in order to quantify the stress distribution, especially for structures with concentric elements such as masses, linear or rotationnal springs. Numerous works have been devoted to the study of the dynamic behaviour of free and forced beam structures carrying one or several masses [1] to [24]. The above-mentioned works have been limited to linear vibrations, which may introduce significant errors especially in the large vibration amplitude domain. A number of works have been devoted to the analysis of large vibration amplitudes of beams with an added mass. Saito et al studied the non-linear dynamic behaviour of a beam with an attached mass subjected to an arbitrarily applied force. The formulation was based on Galerkin's method to get an equation of motion of the Duffing type, solved using the harmonic balance method. The effects of the mass, its position as well as its rotational inertia on the free and forced responses were examined [25]. Using the finite element method, Raju et al analysed the non-linear vibrations of beams with a concentric mass. The effects of the attached mass, its position and magnitude on the non-linear behaviour of the beam were examined for different end conditions and vibration amplitudes [26]. Gutiérrez and Laurra examined the effect of a concentric mass on the large vibration amplitudes of beams and plates using polynomial coordinate functions. According to the author, the algorithm is easily applied to orthotropic plates and can also be applied to beams resting on intermediate supports [27]. Sato et al examined the non-linear vibrations of a beam carrying a point mass with a spring-mass attached system, subjected to a transverse periodic force at an arbitrary point under the influence of gravity. Using Galerkin's method, the non-linear differential equations derived were solved by applying the harmonic balance method [28]. Hamdan and Jubran tackled the free and forced vibrations of a cantilever beam, held at its free end by a translational spring and carrying a concentrated mass arbitrarily located. The beam equation of motion was solved to obtain the mode shape functions, used subsequently in conjunction with Galerkin's method to obtain the beam free and forced response. The effect of the spring stiffness, the concentric mass and its position were examined through a parametric study [29]. Using two perturbation approaches, Pakdemirli and Nayfeh studied the non-linear response of a simply supported beam attached to a spring-mass system at its primary resonance, considering the effects of stretching and damping of the mid-plane of the beam. The first approach consisted on applying the multiple-scale method to the non-linear partial differential equations and end conditions, and the second on averaging the Lagrangian over the fast time scale to obtain the amplitude and phase governing equations. It has been shown that the frequency- 
response and the forced response curves depend on the mid-plane stretching and on the parameters of the spring-mass system [30]. Neglecting the shear strains and the rotational inertia, and considering the axial inertia of the nonlinear curvature and the inextensibility condition, Hamdan and Dado analysed the large free vibration amplitudes of a thin, inextensible cantilever beam with a mass possessing a rotational inertia. A single-mode Lagrangian method was applied to form the third order non-linear unimodal time problem and the two-term harmonic balance (2THB) method was subsequently used to obtain an approximate solution of the oscillation period. The effect of the mass, its position and of the rotational inertia on the oscillation period and its variation with the amplitude was investigated [31]. Applying the multiple-scale method, Özkaya et al studied free and forced nonlinear vibrations of an EulerBernoulli beam with a point mass including the damping effect. The effects of the position and magnitude of the attached mass were identified, as well as the effects of end conditions on the beam dynamic behaviour [32]. Neglecting the shear strains and the rotational inertia, and considering axial inertia and non-linear curvatures, Hamdan and Shabaneh studied the high free vibration amplitudes of a thin, inextensible-cantilevered beam carrying an intermediate mass. Two different approaches were used to formulate the equation of motion, the first used Hamilton's principle to obtain the equation of motion of the partial differential field, and then a single mode approximation was used in conjunction with the Rayleigh-Ritz method to reduce the equation to a non-linear, single mode, Duffing-type time problem. The second approach involved a single mode Lagrangian method that considers the inextensibility condition, and directly forms the unimodal five order nonlinear time problem. Results were illustrated showing the effect of the mass position and the mass magnitude on the variation of the oscillation period with amplitude [33]. Low et al studied experimentally lateral vibrations of an axially constrained beam with a concentrated mass [34]. Karlik et al examined the non-linear vibrations of an Euler-Bernoulli beam carrying a concentrated mass by considering different cases of end conditions. Using the Newton-Raphson method, a parametric study led to determination of the natural frequencies for different mass magnitudes, mass locations and end conditions. Non-linear correction coefficients were calculated using the multiple scale method. The linear and non-linear results were used in training a multi-layer, feed forward, backpropagation artificial neural network algorithm. This algorithm essentially aims to reduce the computation time and allows results to be obtained with an error ranging from 0.5 for linear analysis to 1.5 for non-linear analysis [35]. Using the multi-scale method, Özkaya and Pakdemirli analysed the free and forced nonlinear vibrations of a restrained beam carrying a concentric mass. The perturbation method provided the backbone curves and the nonlinear frequency-response curves [36]. Cheng et al examined the large vibration amplitudes of a cantilever beam carrying a mass and subjected to random base excitation by combining the large amplitude vibration theory of inextensible beams with the stochastic linearization method. The effect of mass and rotational inertia on the beam dynamic behaviour was examined [37]. Considering the damping effect, Özkaya analysed the nonlinear free and forced vibrations of an Euler-Bernoulli beam with concentric masses. Approximate solutions were obtained using the perturbation method, allowing examination of the effect of the added masses, their locations and number on the beam dynamic behaviour [38]. Rao et al studied the largeamplitude vibrations of a uniform Timoshenko beam carrying a centrally concentrated mass using the coupled displacement field method. The results show the effect of the attached mass on the linear and nonlinear behaviour of the beam [39]. Ghayesh et al have developed a general solution procedure using the multiple time scale method for the vibrations of systems with cubic nonlinearities, subjected to nonlinear internal time-dependent boundary conditions [40]. Nikkar et al tackled the large vibration amplitudes of a uniform cantilever beam with an intermediate attached mass using the variational iteration method and He's variational approach to solve the equation of motion. The results showed a good agreement with those obtained by the Range-Kutta fourth order method [41]. Souayeh and Kacem developed a model integrating both geometric and electrostatic nonlinearities for the computation of nonlinear vibrations of electrostatically actuated carbon nanotube-based mass sensors. The Galerkin discretization method was used to reduce the continuous model, solved by the harmonic equilibrium method coupled with the asymptotic numerical method [42]. Barry et al extended the work previously developed by [30] to examine the non-linear vibrations of a beam subjected to an axial force and carrying multiple mass-springdamper systems. The effect of the amplitude and the position of the mass-spring-damper system on the dynamic behaviour of the beam was studied [43]. Considering the effect of an axial force, Malaeke and Moeenfard examined the large free vibration amplitudes of a non-uniform cantilever beam carrying an eccentric mass both transversely and axially. The partial differential equations, obtained using Hamilton's principle, were reduced by a single-mode approximation in conjunction with Lagrange's method and solved using the multiple time-scale perturbation technique [44]. Sadri studied the nonlinear forced vibrations of a cantilever beam with an intermediate lumped mass using Hamilton's principle to obtain the equation of motion and the multiple scales method to solve it. The effects of the damping, the force level and the 
initial conditions on the beam dynamic behaviour were examined [45]. Lotfan and Sadeghi analysed the nonlinear vibrations of a viscoelastic beam described by the Kelvin-Voigt model, and incorporating a mass-spring damper. The nonlinear equations of motion were obtained by Hamilton's principle and solved by the multiple scales method [46]. Rahmouni and Benamar extended the discrete model developed for uniform beams in [?] to study the geometrically nonlinear vibrations of beams with one concentric mass in [47] and with three added masses in [48]. Comparison of the results obtained with those found in the literature established the effectiveness of the discrete model in studying linear and nonlinear vibrations of beams with different types of discontinuities. Bukhari and Barry analysed the nonlinear vibrations of an EulerBernoulli beam carrying a mass-spring system. Hamilton's principle was used to obtain the equations of motion, solved by the multi-scale method [49].

The purpose of the present paper is to investigate the non-linear forced vibrations of beams carrying a concentrated mass under different end conditions. The mathematical model is based on the Euler-Bernoulli beams theory and the von Karman geometrical non-linearity assumptions. Harmonic motion is assumed and the displacement is expanded in the form of a finite series of spatial functions determined by solving the linear problem. Using Hamilton's principle, the problem is reduced to a set of non-linear algebraic equations solved by the so-called second formulation developed in [50]. The non-linear forced deflection shapes and their corresponding curvatures are presented, for different magnitudes of the added mass, different excitation levels and different vibration amplitudes. The objective is to describe the geometrically nonlinear behaviour of a beam with an attached mass and specifically to estimate the nonlinear stress distribution in the case of large transverse vibration amplitudes of the restrained beam excited by a high-level harmonic force.

\section{GENERAL FORMULATION}

In the present study, an Euler-Bernoulli beam with the following geometrical and material characteristics (L, b, h, I, E, A, rho), subjected to transverse vibrations is considered. The beam carries a mass at an arbitrary position $\mathrm{x}$, magnitude $\mathrm{M}$ and inertia $\mathrm{J}$. Two scenarios are considered, in the first one the beam is subjected to a concentric force and in the second to a distributed force. The dynamic behaviour of a conservative system can be obtained by applying the Hamilton's principle that can be written in the form [51]:

$$
\delta \int_{0}^{2 \pi / \omega}\left(\mathbf{V}-\mathbf{T}+\mathbf{W}_{F}\right) d t=0
$$

In which $\mathbf{V}, \mathbf{T}$ and $\mathbf{W}_{F}$ are the beam total strain energy, the kinetic energy and the work done by the excitation force $F$ respectively. Considering large vibration amplitudes, $\mathbf{V}$ can be written as the sum of the axial strain energy $\mathbf{V}_{\mathbf{a}}$ due to the nonlinear stretching forces and the bending strain energy $\mathbf{V}_{\mathbf{b}}$. The expressions of $\mathbf{V}_{\mathbf{a}}, \mathbf{V}_{\mathbf{b}}$ and $\mathbf{T}$ are:

$$
\begin{gathered}
\mathbf{V}_{\mathbf{a}}=\frac{\mathrm{ES}}{8 \mathrm{~L}}\left[\int_{0}^{L}\left(\frac{\partial W(x, t)}{\partial x}\right)^{2} d x\right]^{2} \\
\mathbf{V}_{\mathbf{b}}=\frac{\mathrm{EI}}{2} \int_{0}^{L}\left(\frac{\partial^{2} W(x, t)}{\partial x^{2}}\right)^{2} d x \\
\mathbf{T}=\frac{1}{2} \rho \mathrm{S} \int_{0}^{L}\left(\frac{\partial W(x, t)}{\partial t}\right)^{2} d x \ldots \\
+\frac{1}{2} \sum_{j=1}^{n} \mathbf{M}_{j}\left(\frac{\partial W\left(x_{M}, t\right)}{\partial t}\right)_{x=x_{M}}^{2} \ldots \\
+\frac{1}{2} \sum_{j=1}^{n} \mathbf{J}_{j}\left(\frac{\partial}{\partial t}\left(\frac{\partial W\left(x_{M}, t\right)}{\partial x}\right)\right)_{x=x_{M}}^{2}
\end{gathered}
$$

$\mathrm{W}$ being the transverse displacement of the beam, $M$ the mass magnitude and $J$ the rotational inertia of the attached mass. Assuming harmonic motion and expanding the displacement in the form of a series of functions, the transverse displacement can be written as in [47]:

$$
W(x, t)=a_{i} w_{i} \sin (\omega t)
$$

By replacing $W$ in the expressions for the energies, $\mathbf{V}_{\mathbf{a}}, \mathbf{V}_{\mathbf{b}}, \mathbf{T}$ can be written in the form:

$$
\begin{gathered}
\mathbf{V}_{\mathbf{a}}=\frac{1}{2} a_{i} a_{j} a_{k} a_{l} \mathbf{b}_{\mathrm{ijk}} \sin ^{4}(\omega t) \\
\mathbf{V}_{\mathbf{b}}=\frac{1}{2} a_{i} a_{j} \mathbf{k}_{i j} \sin ^{2}(\omega t) \\
\mathbf{T}=\frac{1}{2} \boldsymbol{\omega}^{2} a_{i} a_{j} \mathbf{m}_{i j} \cos ^{2}(\omega t)
\end{gathered}
$$

Where $\mathbf{k}_{i j}$ denotes the classical rigidity tensor defined by:

$$
\mathbf{k}_{i j}=\int_{0}^{L}\left(\frac{\partial^{2} w_{i}}{\partial x^{2}}\right)\left(\frac{\partial^{2} w_{j}}{\partial x^{2}}\right) d x
$$

$\mathbf{b}_{i j k l}$ the non-linearity tensor given by:

$\mathbf{b}_{i j k l}=\frac{E S}{4 L} \int_{0}^{L}\left(\frac{\partial w_{i}}{\partial x}\right)\left(\frac{\partial w_{j}}{\partial x}\right) d x \int_{0}^{L}\left(\frac{\partial w_{k}}{\partial x}\right)\left(\frac{\partial w_{l}}{\partial x}\right) d x$

$\mathbf{m}_{i j}$ the mass tensor obtained as:

$$
\begin{aligned}
\mathbf{m}_{i j}= & \rho \mathrm{S} \int_{0}^{L} w_{i}(x) w_{j}(x) d x \ldots \\
& +\sum_{i} \mathbf{M}_{j} w_{i}\left(x_{j}\right) w_{j}\left(x_{j}\right) \ldots \\
& +\sum_{i} \mathbf{J}_{j} \boldsymbol{\Psi}_{i}\left(x_{j}\right) \boldsymbol{\Psi}_{j}\left(x_{j}\right)
\end{aligned}
$$

A uniform beam excited by the force $F(x, t)$ over the sub range $S$ of the interval $[0, \mathrm{~L}]$ is considered. The force excites the beam modes via a set of 
generalized forces $F_{i}$ that depend on the expression for $F$, the excitation point for a concentrated excitation, the excitation length for a distributed force and the mode under consideration. The generalized forces $F_{i}(t)$ are given by:

$$
\mathbf{F}_{i}(\mathbf{t})=\int_{\mathrm{S}} F(x, t) w_{i}(x) d x
$$

The non-linear behaviour of the beam is studied first for a point force $F^{c}$ applied at the point $x_{f}$ and then for a distributed harmonic force. The two excitation cases are defined by:

$$
\begin{gathered}
F^{d}(x, t)=f^{d} \sin (\omega t) \\
F^{c}(x, t)=f^{c} \sin (\omega t) \delta\left(x-x_{f}\right)
\end{gathered}
$$

In which $\delta$ is the Dirac function, $F_{i}^{d}(t)$ and $F_{i}^{c}(t)$ are the corresponding generalized forces given by:

$$
\begin{gathered}
F_{i}^{d}(t)=F^{d} \sin (\omega t) \int_{0}^{L} w_{i}(x) d x=f_{i}^{d} \sin (\omega t) \\
F_{i}^{c}(t)=F^{c} \sin (\omega t) w_{i}\left(x_{f}\right)=f_{i}^{c} \sin (\omega t)
\end{gathered}
$$

After calculations, the following non-linear system is obtained:

$$
[\mathbf{K}]\{\mathbf{A}\}+\frac{3}{2}[\mathbf{B}(\{\mathbf{A}\})]\{\mathbf{A}\}-\omega^{2}[\mathbf{M}]\{\mathbf{A}\}=\{\mathbf{F}\}
$$

$\{\mathbf{A}\}$ denotes the column vector of the basic function contribution coefficients. For a nondimensional formulation, one puts:

$$
\begin{gathered}
w_{i}(x)=h w_{i}^{*}\left(\frac{x}{L}\right)=h w_{i}^{*}\left(x^{*}\right) ; \frac{\mathbf{m}_{i j}}{\mathbf{m}_{i j}^{*}}=\rho A h^{2} L \\
\frac{\mathbf{k}_{i j}}{\mathbf{k}_{i j}^{*}}=\frac{E I h^{2}}{L^{3}} ; \frac{\mathbf{b}_{i j k l}}{\mathbf{b}_{i j k l}^{*}}=\frac{E I h^{2}}{L^{3}} ; \frac{\omega^{2}}{\omega^{* 2}}=\frac{E I}{\rho A L^{4}} \\
J_{j}^{*}=\frac{J_{j}}{m L^{3}} \text { and } M_{j}^{*}=\frac{M_{j}}{m L}
\end{gathered}
$$

The dimensionless generalized forces $f_{i}^{* d}$ and

$f_{i}^{* c}$ can be written as:

$$
\begin{gathered}
f_{i}^{* d}=F^{d} \frac{\mathrm{L}^{4}}{\mathrm{EIh}} \int_{0}^{1} w_{i}^{*}\left(x^{*}\right) d x \\
f_{i}^{* c}=F^{c} \frac{\mathrm{L}^{3}}{\mathrm{EIh}} w_{i}^{*}\left(x_{f}\right)
\end{gathered}
$$

After substituting these notations in equation (17), the following nonlinear algebraic system is obtained:

$$
f_{i}^{*^{c}}=F^{c} \frac{\mathrm{L}^{3}}{\operatorname{EIh}} w_{i}^{*}\left(x_{f}\right)
$$

Which may be written in the following tensor form:

$$
\begin{aligned}
& a_{i} \mathbf{k}_{i r}^{*}-\omega^{* 2} a_{i} \mathbf{m}_{i r}^{*}+\frac{3}{2} a_{i} a_{j} a_{k} \mathbf{b}_{i j k r}^{*}=\mathbf{F}_{r}^{*} \quad r=1, \ldots, n \\
& \omega^{* 2} \text { is given as in [52] by: } \\
& \omega^{* 2}=\frac{\{A\}^{T}[K]\{A\}+k\{A\}^{T}[B](\{A\})\{A\}}{\{A\}^{T}[M]\{A\}}
\end{aligned}
$$

With $k=3 / 2$

It has previously been shown that the contribution of one mode remains predominant relatively to the others for the range of amplitudes considered. To indicate that the contributions of the other modes remain small, they are denoted by $\varepsilon_{i}$, while the predominant mode is denoted $a_{r}$.

According to [50], by separating in the non-linear expression $a_{i} a_{j} a_{k} \mathbf{b}_{i j k r}^{*}$ terms proportional to $a_{1}^{3}$, terms proportional to $a_{1}^{2} \varepsilon_{i}$, and by neglecting the terms which are proportional to $a_{1} \varepsilon_{i} \varepsilon_{j}$ and terms proportional to $\varepsilon_{i} \varepsilon_{j} \varepsilon_{k}$ one may write:

$$
a_{i} a_{j} a_{k} \mathbf{b}_{i j k r}=a_{1}^{3} \mathbf{b}_{111 r}+a_{1}^{2} \varepsilon_{i} \mathbf{b}_{11 i r} \quad r=1, \ldots, n
$$

Equation (22) can then be written near the $r^{\text {th }}$ mode as follows:

$$
\begin{aligned}
& \left(\left[\mathbf{K}_{r}^{*}\right]_{R}-\omega^{* 2}\left[\mathbf{M}_{r}^{*}\right]_{R}\right)\left\{\mathbf{A}_{r}\right\}_{R} \\
& +\frac{3}{2}\left[\alpha_{r}^{*}\right]\left\{\mathbf{A}_{r}\right\}_{R}=\left\{\mathbf{F}_{r}-\frac{3}{2} a_{r}^{3} b_{i r r}\right\}
\end{aligned}
$$

With $\left[\alpha_{r}^{*}\right]_{R}=\left[a_{r}^{* 2} b_{i j r r}^{*}\right]_{R}$. Equation (25) is an approximate linear system, very easy to solve in order to get the contribution coefficients to the nonlinear beam forced response.

\section{RESULT AND DISCUSSION}

To validate the presented approach, the results are compared with those obtained by the finite element method developed previously in [26], for two scenarios of boundary conditions: The first table shows results for a fully clamped beam and the second shows the results for a beam simply supported on both sides. The comparison includes also several changes in the mass magnitude and location, and vibration amplitudes. 
Table 1. Comparison of the present results with those obtained by the finite element method for a C-

$\mathrm{C}$ beam carrying a concentrated mass

\begin{tabular}{|c|c|c|c|c|c|c|}
\hline & & & & & & \\
\hline & Mass position & Amplitude & 0.4 & 0.8 & 1 & 2 \\
\hline \multirow{3}{*}{ Reference [26] } & \multirow{3}{*}{$1 / 8$} & 0.2 & 1.0035 & 1.0138 & 1.0215 & 1.0825 \\
\hline & & 0.6 & 1.0034 & 1.0136 & 1.0212 & 1.0811 \\
\hline & & 1 & 1.0034 & 1.014 & 1.0217 & 1.0834 \\
\hline \multirow{3}{*}{ Present } & \multirow{3}{*}{$1 / 8$} & 0.2 & 1.0036 & 1.0142 & 1.0222 & 1.0865 \\
\hline & & 0.6 & 1.0035 & 1.0141 & 1.0219 & 1.0858 \\
\hline & & 1 & 1.0035 & 1.0138 & 1.0216 & 1.0849 \\
\hline \multirow{3}{*}{\multicolumn{2}{|c|}{ Relative difference }} & 0.2 & $0.0072 \%$ & $0.0424 \%$ & $0.0651 \%$ & $0.3701 \%$ \\
\hline & & 0.6 & $0.0127 \%$ & $0.0451 \%$ & $0.0691 \%$ & $0.4313 \%$ \\
\hline & & 1 & $0.0072 \%$ & $0.0148 \%$ & $0.0104 \%$ & $0.1402 \%$ \\
\hline \multirow{3}{*}{ Reference [26] } & \multirow{3}{*}{$1 / 4$} & 0.2 & 1.0035 & 1.014 & 1.0217 & 1.0834 \\
\hline & & 0.6 & 1.0036 & 1.0143 & 1.0222 & 1.0856 \\
\hline & & 1 & 1.0037 & 1.0147 & 1.0228 & 1.0881 \\
\hline \multirow{3}{*}{ Present } & \multirow{3}{*}{$1 / 4$} & 0.2 & 1.0035 & 1.0141 & 1.0220 & 1.0862 \\
\hline & & 0.6 & 1.0034 & 1.0137 & 1.0214 & 1.0846 \\
\hline & & 1 & 1.0033 & 1.0133 & 1.0208 & 1.0830 \\
\hline \multirow{3}{*}{\multicolumn{2}{|c|}{ Relative difference }} & 0.2 & $0.0045 \%$ & $0.0128 \%$ & $0.0313 \%$ & $0.2612 \%$ \\
\hline & & 0.6 & $0.0170 \%$ & $0.0597 \%$ & $0.0812 \%$ & $0.0940 \%$ \\
\hline & & 1 & $0.0366 \%$ & $0.1353 \%$ & $0.1938 \%$ & $0.4661 \%$ \\
\hline \multirow{3}{*}{ Reference [26] } & \multirow{3}{*}{$3 / 8$} & 0.2 & 1.0036 & 1.0142 & 1.022 & 1.0844 \\
\hline & & 0.6 & 1.0036 & 1.0144 & 1.0224 & 1.086 \\
\hline & & 1 & 1.0037 & 1.0145 & 1.0226 & 1.0869 \\
\hline \multirow{3}{*}{ Present } & \multirow{3}{*}{$3 / 8$} & 0.2 & 1.0036 & 1.0143 & 1.0223 & 1.0869 \\
\hline & & 0.6 & 1.0036 & 1.0142 & 1.0221 & 1.0862 \\
\hline & & 1 & 1.0035 & 1.0141 & 1.0219 & 1.0858 \\
\hline \multirow{3}{*}{\multicolumn{2}{|c|}{ Relative difference }} & 0.2 & $0.0007 \%$ & $0.0108 \%$ & $0.0282 \%$ & $0.2300 \%$ \\
\hline & & 0.6 & $0.0040 \%$ & $0.0216 \%$ & $0.0303 \%$ & $0.0218 \%$ \\
\hline & & 1 & $0.0164 \%$ & $0.0406 \%$ & $0.0637 \%$ & $0.1055 \%$ \\
\hline \multirow{3}{*}{ Reference [26] } & \multirow{3}{*}{$1 / 2$} & 0.2 & 1.0035 & 1.0138 & 1.0215 & 1.0823 \\
\hline & & 0.6 & 1.0035 & 1.0137 & 1.0213 & 1.0816 \\
\hline & & 1 & 1.0034 & 1.0137 & 1.0213 & 1.0814 \\
\hline \multirow{3}{*}{ Present } & \multirow{3}{*}{$1 / 2$} & 0.2 & 1.0036 & 1.0144 & 1.0223 & 1.0868 \\
\hline & & 0.6 & 1.0036 & 1.0144 & 1.0223 & 1.0867 \\
\hline & & 1 & 1.0036 & 1.0143 & 1.0223 & 1.0866 \\
\hline \multirow{3}{*}{\multicolumn{2}{|c|}{ Relative difference }} & 0.2 & $0.0106 \%$ & $0.0547 \%$ & $0.0828 \%$ & $0.4166 \%$ \\
\hline & & 0.6 & $0.0107 \%$ & $0.0647 \%$ & $0.1022 \%$ & $0.4684 \%$ \\
\hline & & 1 & $0.0205 \%$ & $0.0637 \%$ & $0.1006 \%$ & $0.4786 \%$ \\
\hline
\end{tabular}


Table 2. Comparison of the present results with those obtained by the finite element method for a

\begin{tabular}{|c|c|c|c|c|c|c|}
\hline & & & \multicolumn{4}{|c|}{ S-S beam carrying a concentrated mass } \\
\hline & Mass position & Amplitude & 0.4 & 0.8 & 1 & 2 \\
\hline \multirow{3}{*}{ Reference [26] } & \multirow{3}{*}{$1 / 8$} & 0.2 & 1.015 & 1.0584 & 1.0897 & 1.3157 \\
\hline & & 0.6 & 1.0153 & 1.0595 & 1.0914 & 1.3226 \\
\hline & & 1 & 1.0155 & 1.0604 & 1.0928 & 1.3287 \\
\hline \multirow{3}{*}{ Present } & \multirow{3}{*}{$1 / 8$} & 0.2 & 1.0149 & 1.0585 & 1.0900 & 1.3244 \\
\hline & & 0.6 & 1.0149 & 1.0586 & 1.0902 & 1.3276 \\
\hline & & 1 & 1.0149 & 1.0585 & 1.0903 & 1.3309 \\
\hline & \multirow{3}{*}{ Relative difference } & 0.2 & $0.0075 \%$ & $0.0052 \%$ & $0.0260 \%$ & $0.6586 \%$ \\
\hline & & 0.6 & $0.0365 \%$ & $0.0887 \%$ & $0.1067 \%$ & $0.3768 \%$ \\
\hline & & 1 & $0.0609 \%$ & $0.1804 \%$ & $0.2332 \%$ & $0.1687 \%$ \\
\hline \multirow{3}{*}{ Reference [26] } & \multirow{3}{*}{$1 / 4$} & 0.2 & 1.015 & 1.0587 & 1.09 & 1.3173 \\
\hline & & 0.6 & 1.0153 & 1.0596 & 1.0916 & 1.3243 \\
\hline & & 1 & 1.0154 & 1.0602 & 1.0925 & 1.3286 \\
\hline \multirow{3}{*}{ Present } & \multirow{3}{*}{$1 / 4$} & 0.2 & 1.0149 & 1.0584 & 1.0900 & 1.3251 \\
\hline & & 0.6 & 1.0148 & 1.0582 & 1.0898 & 1.3281 \\
\hline & & 1 & 1.0147 & 1.0578 & 1.0893 & 1.3298 \\
\hline \multirow{3}{*}{\multicolumn{2}{|c|}{ Relative difference }} & 0.2 & $0.0086 \%$ & $0.0246 \%$ & $0.0008 \%$ & $0.5937 \%$ \\
\hline & & 0.6 & $0.0482 \%$ & $0.1332 \%$ & $0.1680 \%$ & $0.2894 \%$ \\
\hline & & 1 & $0.0703 \%$ & $0.2239 \%$ & $0.2891 \%$ & $0.0899 \%$ \\
\hline \multirow{3}{*}{ Reference [26] } & \multirow{3}{*}{$3 / 8$} & 0.2 & 1.0147 & 1.0574 & 1.088 & 1.3089 \\
\hline & & 0.6 & 1.0146 & 1.0568 & 1.0871 & 1.3055 \\
\hline & & 1 & 1.0145 & 1.0565 & 1.0866 & 1.3036 \\
\hline \multirow{3}{*}{ Present } & \multirow{3}{*}{$3 / 8$} & 0.2 & 1.0148 & 1.0580 & 1.0893 & 1.3218 \\
\hline & & 0.6 & 1.0147 & 1.0576 & 1.0886 & 1.3204 \\
\hline & & 1 & 1.0146 & 1.0572 & 1.0882 & 1.3196 \\
\hline \multirow{3}{*}{\multicolumn{2}{|c|}{ Relative difference }} & 0.2 & $0.0118 \%$ & $0.0609 \%$ & $0.1235 \%$ & $0.9855 \%$ \\
\hline & & 0.6 & $0.0080 \%$ & $0.0710 \%$ & $0.1415 \%$ & $1.1430 \%$ \\
\hline & & 1 & $0.0088 \%$ & $0.0690 \%$ & $0.1457 \%$ & $1.2294 \%$ \\
\hline \multirow{3}{*}{ Reference [26] } & \multirow{3}{*}{$1 / 2$} & 0.2 & 1.0145 & 1.0564 & 1.0865 & 1.3026 \\
\hline & & 0.6 & 1.0142 & 1.0551 & 1.0844 & 1.2940 \\
\hline & & 1 & 1.0140 & 1.0545 & 1.0834 & 1.2900 \\
\hline \multirow{3}{*}{ Present } & \multirow{3}{*}{$1 / 2$} & 0.2 & 1.0147 & 1.0577 & 1.0888 & 1.3191 \\
\hline & & 0.6 & 1.0146 & 1.0572 & 1.0880 & 1.3161 \\
\hline & & 1 & 1.0145 & 1.0569 & 1.0875 & 1.3148 \\
\hline \multirow{3}{*}{\multicolumn{2}{|c|}{ Relative difference }} & 0.2 & $0.0244 \%$ & $0.1261 \%$ & $0.2146 \%$ & $1.2702 \%$ \\
\hline & & 0.6 & $0.0393 \%$ & $0.1948 \%$ & $0.3274 \%$ & $1.7082 \%$ \\
\hline & & 1 & $0.0516 \%$ & $0.2246 \%$ & $0.3803 \%$ & $1.9218 \%$ \\
\hline
\end{tabular}

Tables 1 and 2 show a very good agreement between the results of the present method and those of the literature, since the relative difference varies from 0.02 to $0.47 \%$ in the case of a fully clamped beam and from 0.05 to $1.92 \%$ in the case of a simply supported beam, for a maximum nondimensional amplitude "Wmax=2".

The analysis of the forced case via the method mentioned above requires determination of the mode predominant in the beam response 
corresponding to the level and location of the excitation force. Table 3 gives the numercal values of the generalized forces corresponding to the different modes for a beam excited at its centre, for three different excitation levels.

Table 3. Percentage of generalised forces exciting the first five symmetric modes of a Clamped-Clamped beam carrying

\begin{tabular}{|r|r|r|r|r|r|r|}
\hline & \multicolumn{9}{|c|}{ Modes } & 1 & 3 & 5 & 7 & 9 \\
\hline \multirow{2}{*}{$\mathrm{F} c=10$} & $F^{c} w_{i}^{*}\left(x_{f}\right)$ & 10.65 & 5.32 & 3.87 & 2.99 & 2.43 \\
\cline { 2 - 7 } & $F^{c} w_{i}^{*}\left(x_{f}\right) / \sum_{i=1}^{n} \mid F^{c} w_{i}^{*}\left(x_{f}\right)$ & 42.14 & 21.08 & 15.31 & 11.84 & 9.63 \\
\hline \multirow{2}{*}{$\mathrm{Fc}=100$} & $F^{c} w_{i}^{*}\left(x_{f}\right)$ & 106,49 & 53,26 & 38,69 & 29,91 & 24,32 \\
\cline { 2 - 7 } & $F^{c} w_{i}^{*}\left(x_{f}\right) / \sum_{i=1}^{n}\left|F^{c} w_{i}^{*}\left(x_{f}\right)\right|$ & 42.14 & 21.08 & 15.31 & 11.84 & 9.63 \\
\hline \multirow{2}{*}{$\mathrm{Fc}=200$} & $F^{c} w_{i}^{*}\left(x_{f}\right)$ & 212,98 & 106,53 & 77,38 & 59.82 & 48.65 \\
\cline { 2 - 7 } & $F^{c} w_{i}^{*}\left(x_{f}\right) / \sum_{i=1}^{n} \mid F^{c} w_{i}^{*}\left(x_{f}\right)$ & 42.14 & 21.08 & 15.31 & 11.84 & 9.63 \\
\hline
\end{tabular}

It appears clearly from Table 3, that, for the beam considered, the first mode remains predominant for the three excitation levels considered. The study of the forced case will then focus on the solution of the system in the vicinity of the first mode as described in the general formulation presented above.

The curvatures associated to the non-linear forced response are shown in Figure1 for different magnitudes of the concentric mass $\left(\mathrm{m}^{*}=0.25\right.$, $\left.\mathrm{m}^{*}=0.5, \mathrm{~m}^{*}=1\right)$, for the same maximum nondimensional amplitude "Wmax $=1.5$ " and for a concentric force applied at the beam centre, with intensity corresponding to " $\mathrm{fc}=100$ ". It can be seen that as the magnitude of the mass increases, the stress also increases at the beam centre were the mass is attached, but decreases near to the clamps. It should also be noted in table 4, that the nonlinearity effect becomes more pronounced in the middle of the beam by increasing the magnitude of the mass. The percentage correction introduced by the non-linear theory compared to the linear theory increases from $1.06 \%$ for a mass of 0.25 to $5.32 \%$ for a mass of 0.5 , and to $9.09 \%$ for a mass of magnitude equal to 1 . On the other hand, a decrease of the effect can be noticed in the vicinity of the clamps. Figure 2 show the non-linear forced curvatures for different levels of excitation $(\mathrm{fc}=0$, $f c=100$, $f c=200)$, for the same maximum nondimensional amplitude "Wmax $=2$ " and for a mass attached at the middle of the beam, with a magnitude $" m^{*}=0.5 "$. It can be observed that the concentric force increases the stress at the beam middle (where the mass is attached), but decreases it near to the clamps. It is clearly shown that the stress variation is not proportional to the force level as the principle of superposition applied in the linear domain is invalid at large vibration amplitudes. It should be noted in table 5, that by increasing the intensity of the force the effect of non-linearity increases near the clamps but decreases in the middle. For example, for a free beam, the correction percentage is estimated at $24.83 \%$ near the recesses but is less at the middle of the beam with $10.32 \%$. The correction percentage increases afterwards by applying a force of $100 \mathrm{~N}$ at 25.54 near the clamps but decreases to 9.51 at the beam centre. The non-linear forced vibration deflections shapes and their associated curvatures are respectively plotted in figures 5 and 6 for different amplitudes of vibration (Wmax $=0.8$, $\mathrm{W} \max =1$, $\mathrm{W} \max =1.5$, $\mathrm{Wmax}=2$ ), for the same mass magnitude " $\mathrm{m} *=0.5$ " and for a concentric force applied at the middle of the beam, with an intensity: " $\mathrm{fc}=100$ ". It is clear that the amplitude of vibration increases considerably the stresses at both the mass location and near to the clamps, which constituted the main motivation for the present study. In table 6 , it seems very clear that the effect of non-linearity increases considerably by increasing the vibration amplitude either in the middle of the beam or in the vicinity of the clamps. In the vicinity of the clamps, the percentage of correction introduced by the non-linear theory compared to the linear theory increases for example from $6.05 \%$ for a maximum non-dimensional amplitude of 0.8 to $25.85 \%$ for a maximum nondimensional amplitude of 2 . The percentage also increases in the middle of the beam but is less pronounced since it varies only from $1.35 \%$ to $9.51 \%$ for the same example. 


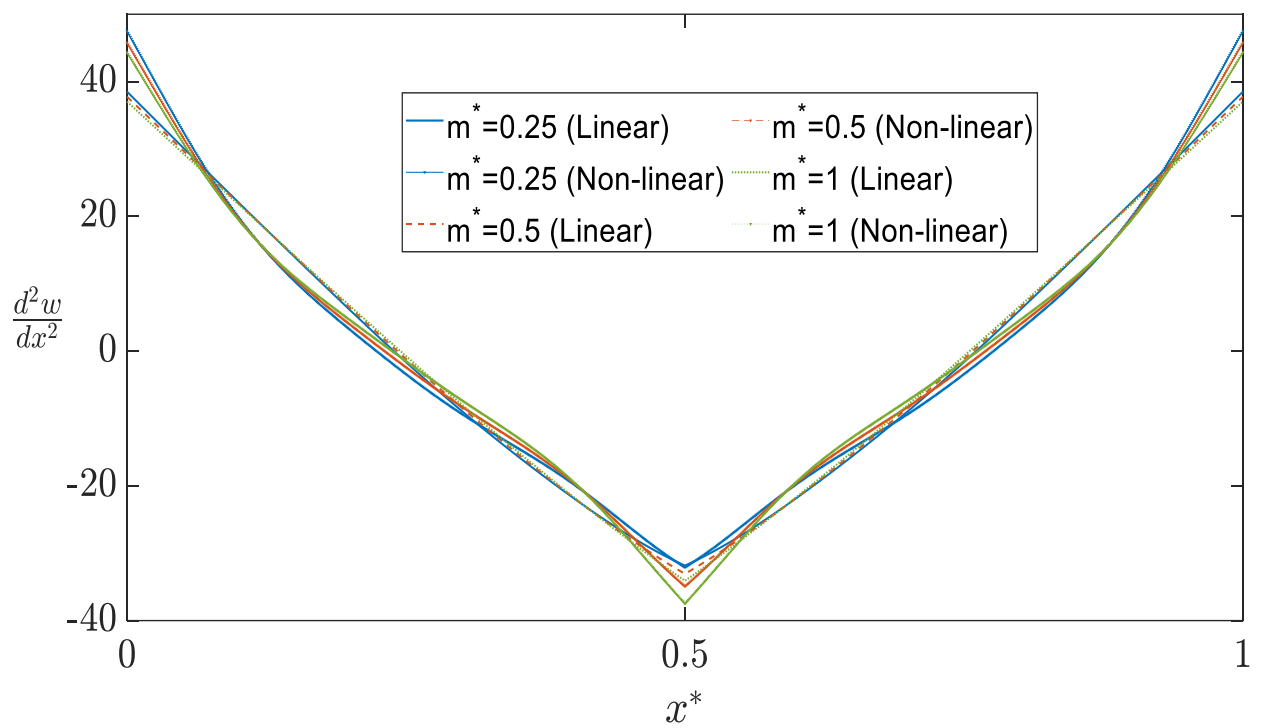

Figure 1. Curvatures corresponding to the non-linear response deflection shapes for various mass magnitudes.

Table 4. Effect of mass variation on the percentage correction introduced by the non-linear theory compared to the linear

\begin{tabular}{|r|c|c|c|}
\hline Mass & 0.25 & 0.5 & 1 \\
\hline Clamps & 18.97 & 17.53 & 16.44 \\
\hline Middle of the beam & 1.06 & 5.32 & 9.09 \\
\hline
\end{tabular}

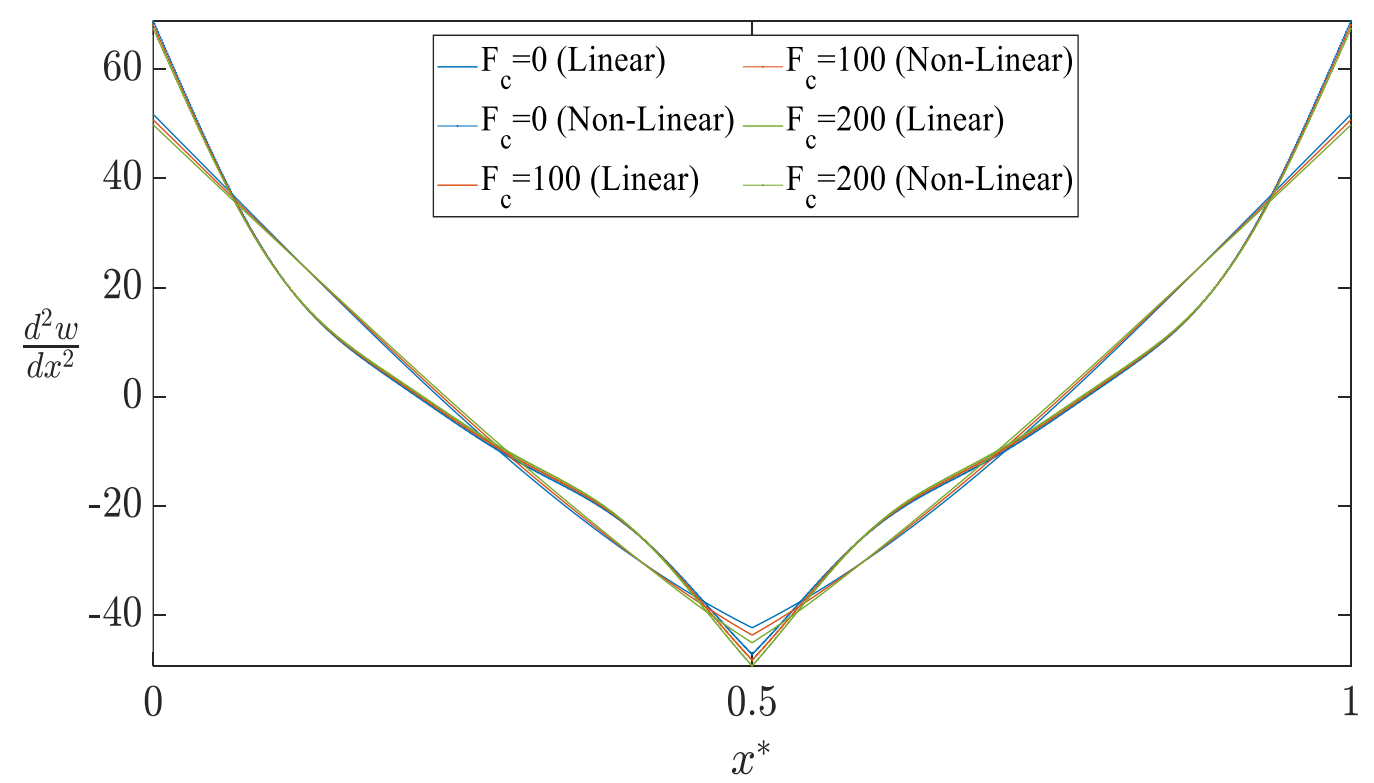

Figure 2. Curvatures corresponding to the non-linear deflection response function for various excitation levels

Table 5. Effect of force variation on the percentage correction introduced by the non-linear theory compared to the linear

\begin{tabular}{|r|r|r|r|}
\multicolumn{2}{|c|}{ theory } \\
\hline Force & 0 & 100 & 200 \\
\hline Clamps & 24.83 & 25.54 & 26.13 \\
\hline Middle of the beam & 10.32 & 9.51 & 8.63 \\
\hline
\end{tabular}




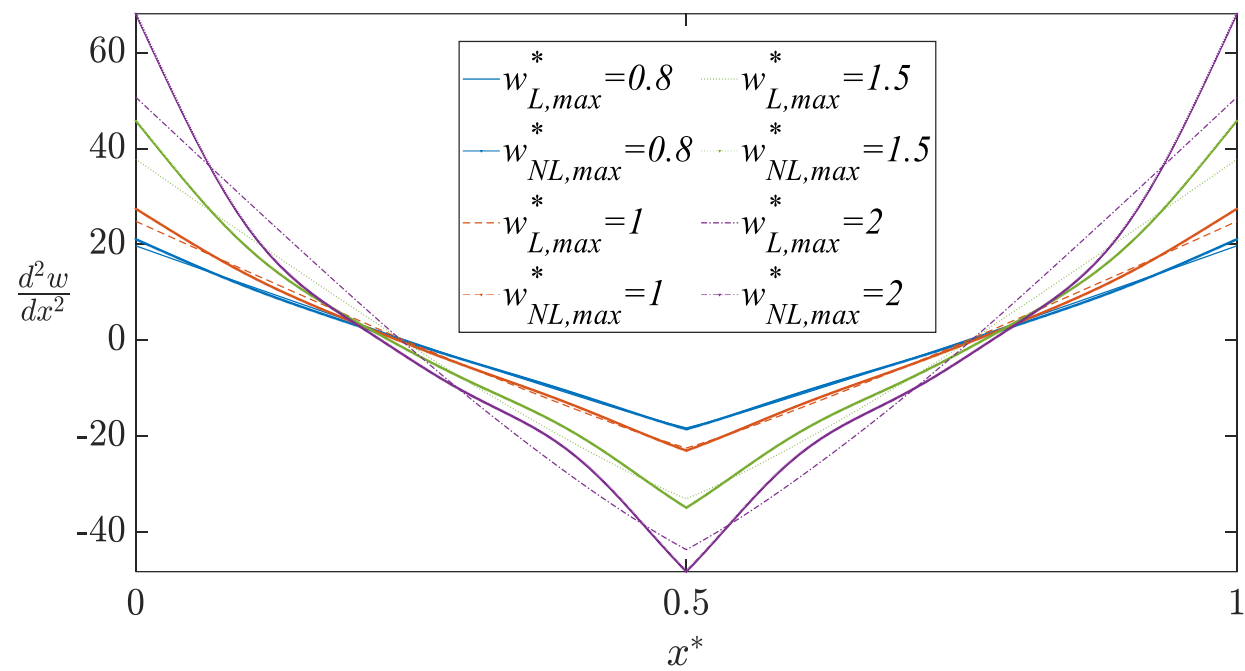

Figure 3. Curvatures corresponding to the non-linear response deflection shapes for various amplitudes

Table 6. Effect of mass variation on the percentage correction introduced by the non-linear theory compared to the linear

\begin{tabular}{|r|r|r|r|r|}
\multicolumn{2}{|c|}{ theory } \\
\hline Amplitudes & 0.8 & 1 & 1.5 & 2 \\
\hline Clamps & 6.05 & 9.51 & 17.53 & 25.85 \\
\hline Middle of the beam & 1.35 & 2.22 & 5.32 & 9.51 \\
\hline
\end{tabular}

\section{CONCLUSIONS}

The geometrically non-linear forced vibrations of a uniform beam carrying a concentrated mass were examined. The expressions for the kinetic and potential energies were derived and, by applying Hamilton's principle, the problem was reduced to a non-linear algebraic system solved by an approximate method. The results were then compared with those obtained by the finite element method and showed a very good agreement since the relative difference does not exceed $0.47 \%$ for the case of a fully clamped beam and does not exceed $1.92 \%$ for a beam with simple supports. The forced non-linear mode shapes and their associated curvatures were illustrated for different types of excitations, different magnitudes of the attached mass, different levels of excitation and different amplitudes. It was noticed that the stresses increase by increasing the magnitude of the mass and decrease near to the clamps. It was clearly noticed that the greater the amplitude, the greater the distribution of stresses for the same beam considered, carrying the same mass and subjected to the same excitation. The analysis also led to a quantitative estimate of the percentage correction introduced by the non-linear theory compared to the linear theory.

\section{REFERENCES}

1. Maltbaek JC. The influence of a concentrated mass on the free vibrations of a uniform beam. J. Mech. Sci. 1961; 3:197-218.

2. Chen Y. On the vibration of beams or rods carrying a concentrated mass. J. Appl. Mech. 1963; 30(2):310.
3. Srinath LS, Das YC. Vibrations of beams carrying mass. J. Appl. Mech. Trans. ASME. 1967. 34(3): 784-785,

4. Laura PAA, Pombo JL, Susemihl EA. A note on the vibrations of a clamped-free beam with a mass at the free end. J. Sound Vib. 1974; 37(2): 161-168.

5. Laura PAA, Maurizi MJ, Pombo JL. A note on the dynamic analysis of an elastically restrained-free beam with a mass at the free end. J. Sound Vib. 1975;41:397-405.

6. Laura PAA, Verniere de Irassar P, Ficcadent GM. A note on transverse vibrations of continuous beams subject to an axial force and carrying concentrated masses. J. Sound Vib. 1983; 86: 279-284.

7. Gurgoze M. A note on the vibrations of restrained beams and rods with point masses. J. Sound Vib. 1984; 9:461-468.

8. Liu WH, Wu JR, Huang CC. Free vibration of beams with elastically restrained edges and intermediate concentrated masses. J. Sound Vib. 1988; 122(2):193-207.

9. Wu JS, Lin TL. Free vibration analysis of a uniform cantilever beam with point masses by an analyticaland-numerical-combined method. J. Sound Vib. 1990;136(2):201-213.

10. Leckie F, Pestel E. Transfer-matrix fundamentals. Int. J. Mech. Sci. 1960; 2(3):137-167.

11. Hamdan M, Abdellatif L. On the numerical convergence of discretization methods for the free vibrations of beams with attached inertia elements. J. Sound Vib. 1994.

12. Gurgoze M. On the alternative formulations of the frequency equation of a Bernoulli-Euler beam to which several spring-mass systems are attached inspan. Development. 1998;134(4): 635-646.

13. Wu JS, Chou HM. A new approach for determining the natural frequencies and mode shapes of a uniform beam carrying any number of sprung masses. J. Sound Vib. 1999;220(3):451-468.

14. Chang $\mathrm{CH}$. Free vibration of a simply supported beam carrying a rigid mass at the middle. J. Sound Vib. 2000;237;4:733-744. 
15. Naguleswaran S. Transverse vibrations of an EulerBernoulli uniform beam carrying two particles inspan. Int. J. Mech. Sci. 2001;43: 2737-2752.

16. Naguleswaran S. Transverse vibration of an Euler Bernoulli uniform beam on up to five resilient supports including ends. J. Sound Vib. 2003;261: 372-384.

17. Lin HY, Tsai YC. Free vibration analysis of a uniform multi-span beam carrying multiple springmass systems. J. Sound Vib. 2007;302(3): 442-456. https://doi.org/10.1016/i.jsv.2006.06.080

18. Maiz S, Bambill DV, Rossit CA, Laura PAA. Transverse vibration of Bernoulli-Euler beams carrying point masses and taking into account their rotatory inertia: Exact solution. J. Sound Vib. 2007; 303(3-5):895-908.

https://doi.org/10.1016/j.jsv.2006.12.028

19. Wang J, Qiao P. Vibration of beams with arbitrary discontinuities and boundary conditions. J. Sound Vib. 2007;308(1-2):12-27. https://doi.org/10.1016/j.jsv.2007.06.071

20. Yesilce Y, Demirdag O, Catal S. Free vibrations of a multi-span Timoshenko beam carrying multiple spring-mass systems. Sadhana. 2008;33: 385-401.

21. Yesilce Y, Demirdag O. Effect of axial force on free vibration of Timoshenko multi-span beam carrying multiple spring-mass systems. Int. J. Mech. Sci. 2008;50:995-1003.

https://doi.org/10.1016/j.ijmecsci.2008.03.001

22. Yesilce Y. Effect of axial force on the free vibration of reddy-bickford multi-span beam carrying multiple spring-mass systems. Shock Vib. 2008; 18(5):709726. https://doi.org/10.1177/1077546309102673

23. Torabi K, Afshari H, Najafi H. Vibration analysis of multi-step Bernoulli-Euler and Timoshenko beams carrying concentrated masses. J. Solid Mech. 2013; 5(4):336-349.

24. Yesilce Y. Differential transform method and numerical assembly technique for free vibration analysis of the axial-loaded Timoshenk. Struct. Eng. Mech. 2015.

25. Saito H, Sato K, Yutani T. Non-linear forced vibrations of a beam carrying concentrated mass under gravity. J. Sound Vib. 1976; 46(4): 515-525.

26. Raju KK, Rao GV. Non-linear vibrations of beams carrying a concentrated mass. J. Sound Vib. 1976; 48(3):445.

27. Gutiérrez RH, Laura PAA. Effect of a concentrated mass on large amplitude, free flexural vibrations of elastic plates and beams. Appl. Acoust. 1984; 17(2): 135-151.

28. Kiichi S, Genji S, Yoshiya M. Non-linear vibrations of beams with attached spring-mass system. Japan Soc. Mech. Eng. 1986;29:2232-2238.

29. Hamdan MN, Jubran BA. Free and forced vibrations of a restrained cantilever beam carrying a concentrated mass. J. King Abdulaziz Univ. Sci. 1991; 3(1):71-83.

30. Pakdemirli M, Nayfeh AH. Nonlinear vibrations of a beam- spring-mass system. J. Vib. Acoust. 1994; 116:433-439.

31. Hamdan MN, Dado MHF. Large amplitude free vibrations of a uniform cantilever beam carrying an intermediate lumped mass and rotary inertia. J. Sound Vib. 1997; 206(2):151-168.

32. Özkaya E, Pakdemirli M, Öz HR. Non-linear vibrations of a beam-mass system under different boundary conditions. J. Sound Vib. 1997; 199(4): 679-696.

33. Hamdan MN, Shabaneh NH. On the large amplitude free vibrations of a restrained uniform beam carrying an intermediate lumped mass. J. Sound Vib. 1997; 199(5): 711-736.

34. Low KH, Dubey RN. Experimental investigations and shape functions for lateral vibration of axially constrained beams with a concentrated mass at the centre. J. Sound Vib. 1997; 202(3): 446-451.

35. Karlik B, Özkaya E, Aydin S, Pakdemirli M. Vibrations of a beam-mass systems using artificial neural networks. Comput. Struct. 1998; 69(3): 339347.

36. Pakdemirli M, Ozkaya E. Non-linear vibrations of a beam-mass system with both ends clamped. J. Sound Vib. 1999; 221(3): 491-503.

37. Cheng G, Mei C, Lee RYY. Large amplitude vibration of a cantilever beam with tip mass under random base excitation. Adv. Struct. Eng. 2001; 4(4):203-210.

38. Özkaya E. Non-linear transverse vibrations of a simply supported beam carrying concentrated masses. J. Sound Vib. 2002; 257(3):413-424. https://doi.org/10.1006/jsvi.2002.5042

39. G. Venkateswara Rao, K. Meera Saheb, and G. Ranga Janardhan, "Fundamental frequency for large amplitude vibrations of uniform Timoshenko beams with central point concentrated mass using coupled displacement field method," J. Sound Vib., vol. 298, no. 1-2, pp. 221-232, 2006.

40. Ghayesh MH, Kazemirad S, Darabi MA. A general solution procedure for vibrations of systems with cubic nonlinearities and nonlinear/time-dependent internal boundary conditions. J. Sound Vib. 2011; 330(22):5382-5400. https://doi.org/10.1016/j.jsv.2011.06.001

41. NikkarA, Bagheri S, Saravi M. Dynamic model of large amplitude vibration of a uniform cantilever beam carrying an intermediate lumped mass and rotary inertia. Lat. Am. J. Solids Struct. 2014;11(2):320-329. https://doi.org/10.1590/S167978252014000200010.

42. Souayeh S, Kacem N. Computational models for large amplitude nonlinear vibrations of electrostatically actuated carbon nanotube-based mass sensors. Sensors Actuators A Phys.2014:208: 10-20. https://doi.org/10.1016/j.sna.2013.12.015

43. Barry OR, Oguamanam DCD, Zu JW. Nonlinear vibration of an axially loaded beam carrying multiple mass-spring-damper systems. Nonlinear Dyn. 2014; 77(4):1597-1608. https://doi.org/10.1007/s11071-014-1402-5

44. Malaeke H, Moeenfard H. Analytical modeling of large amplitude free vibration of non-uniform beams carrying a both transversely and axially eccentric tip mass. J. Sound Vib. 2016; 366:211-229. https://doi.org/10.1016/j.jsv.2015.12.003

45. Sadri M, Younesian D, Esmailzadeh E. Nonlinear harmonic vibration and stability analysis of a cantilever beam carrying an intermediate lumped mass. Nonlinear Dyn. 2016; 84; 3:1667-1682. https://doi.org/10.1007/s11071-016-2596-5

46. Lotfan S, Sadeghi MH. Large amplitude free vibration of a viscoelastic beam carrying a lumped mass-spring-damper. Nonlinear Dyn. 2017;90(2): 1053-1075. https://doi.org/10.1007/s11071-017$\underline{3710-\mathrm{z}}$ 
47. Rahmouni A, Benamar R. A discrete model for geometrically non-linear transverse free constrained vibrations of beams carrying a concentrated mass at various locations. Proc. Int. Conf. Struct. Dyn. 2014:2093-2099.

48. Rahmouni A, Benamar R. Nonlinear transverse vibrations of clamped beams carrying two or three concentrated masses at various locations. MATEC Web Conf. 2016; 83:1-4.

https://doi.org/10.1051/matecconf/20168305009

49. Bukhari MA, Barry OR. Nonlinear vibrations of a beam- spring-large mass system. Proc. ASME 2017 Int. Mech. Eng. Congr. Expo. IMECE2017. 2017: $1-7$.

50. El Kadiri M, Benamar R, White RG. Improvement of the semi-analytical method, for determining the geometrically non-linear response of thin straight structures. Part I: Application to clamped-clamped and simply supported-clamped beams. J. Sound Vib. 2002;249(2):263-305.

51. Géradin M, Rixen DJ. Mechanical vibrations: theory and application to structural dynamics. 3rd Editio. 2015.

52. El Bikri K, Benamar R, Bennouna MM. Geometrically non-linear free vibrations of clampedclamped beams with an edge crack. Comput. Struct. 2006;84(7):485-502.

\section{Received 2020-07-19}

Accepted 2020-10-19

Available online 2020-11-09
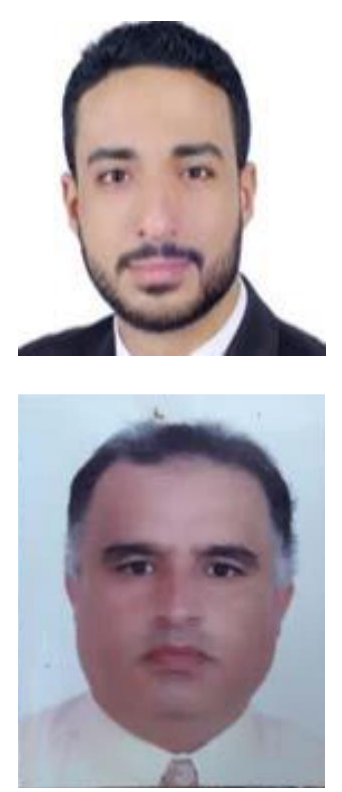

Ahmed ADRI - Professor of Mechanical Engineering at the Superior School of Technology (EST) University Hassan II of Casablanca, Morocco. He got his engineering degree in Design and Mechanical Manufacturing from the Mohammadia School of Engineers (EMI) - Mohammed V University of Rabat, Morocco.s

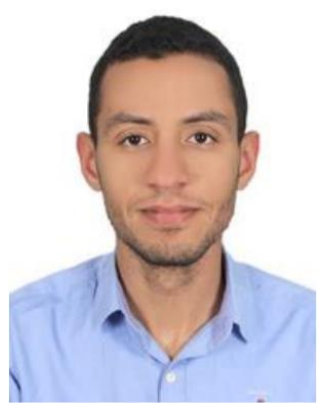

Mohcine CHAJDI - PhD student in the research team "Modeling Structures and Mechanical Systems" (M2SM) and holder of a Master's degree in Mechanical Engineering in 2015 at Higher School of Technical Education (ENSET) Mohammed $\mathrm{V}$ University of Rabat, Morocco.

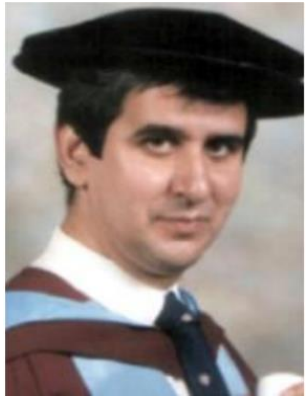

Rhali BENAMAR - Professor of Mechanical Engineering and Vibrations at the Mohammadia School of Engineering (EMI) Mohammed V University of Rabat, Morocco. He got his engineering degree in civil engineering in 1982 from the ${ }^{3}$ Ecole Nationale des Ponts et Chaussées" of Paris, France. He received his Ph.D. in 1990 from the Institute of Sound and Vibration at the University of Southampton, United Kingdom. 